\title{
Cerebral correlates of explicit sequence learning
}

Destrebecqz Arnaud[1], Peigneux Philippe [2][3], Laureys Steven[2][4], Degueldre Christian[2], Del Fiore Guy[2], Aerts Joel[2], Luxen André[2] , van der Linden Martial[3], Cleeremans Axel[1], Maquet Pierre[2][4][5]

[1] Cognitive Science Research Unit, Universite Libre de Bruxelles, Bruxelles, Belgium

[2] Cyclotron Research Center, University of Liège, Liège, Belgium

[3] Department of Neuropsychology, University of Liège, Liège, Belgium

[4]Department of Neurology, CHU Sart, Tilman, Belgium

[5]Wellcome Department of Cognitive Neurology, University College London, London, UK

\begin{abstract}
Using positron emission tomography (PET) and regional cerebral blood flow (rCBF) measurements, we investigated the cerebral correlates of consciousness in a sequence learning task through a novel application of the Process Dissociation Procedure, a behavioral paradigm that makes it possible to separately assess conscious and unconscious contributions to performance. Results show that the metabolic response in the anterior cingulate/mesial prefrontal cortex (ACC/MPFC) is exclusively and specifically correlated with the explicit component of performance during recollection of a learned sequence. This suggests a significant role for the ACC/MPFC in the explicit processing of sequential material.
\end{abstract}

Keywords: Implicit and explicit sequence learning; PET; Anterior cingulate cortex; Mesial prefrontal cortex

\section{Introduction}

The relationship between learning and consciousness is a central issue in the neurosciences [14] and in cognitive psychology [37]. While both learning [8] and memory [19] can occur without awareness, considerable debate continues about how to best establish anatomical and functional differences between implicit and explicit processes.

Sequence learning has now become the main behavioral paradigm through which to study learning without awareness (i.e., implicit learning). Many reports have shown that subjects can learn the regularities of a structured sequence of stimuli when faced with a speeded sequential reaction time (SRT) task in which they simply have to press as fast and as accurately as possible on the key corresponding to the location of a stimulus displayed on a computer screen [10]. Interestingly, while participants learn the transitions of the repeated sequence, as demonstrated by dramatic increases in reaction time when the sequence is modified in any way [31], they often fail to be able to describe the sequential regularities contained in the material when probed to do so-a result that suggests that sequence learning was implicit. Other studies have shown, however, that participants, in general, are able to deploy their knowledge of the training sequence intentionally onto subsequent sequence generation or sequence recognition tasks, in which they are respectively instructed to reproduce the sequential pattern they have been exposed to, or to classify small sequence fragments as instances of the training material or not [36]. These results are usually taken as evidence for explicit rather than implicit sequence learning. A recent study has shown, however, that generation performance may be primarily based on implicit or explicit knowledge depending on the training conditions in the SRT task [12]. This latter result therefore suggests that learning in the SRT task may also depend on both explicit and implicit processes.

Previous sequence learning studies using neuroimaging methods have reported that implicit and explicit learning processes involve distinct areas of the brain $[15,30]$. However, the brain regions that were associated with implicit and explicit processes only partially overlapped between these studies. One possible reason for this discrepancy may be that explicit and implicit learning processes were 
associated either with different training conditions, or with different training phases. The cerebral regions that are specifically linked to either process therefore remain uncertain.

Moreover, these and other behavioral studies have tended to use absolute measures of awareness. Such measures, however, are not immune from possible contamination by implicit influences. Indeed, in the absence of a clear operational criterion for awareness, it appears premature to consider that there exist tasks that exclusively involve either conscious or unconscious processes. In a memory task, for instance, after studying a list of words, participants may use these words in a stem completion task either because they recollect them explicitly, or simply because of a feeling of familiarity that may not be associated with conscious recollection-a distinction between recollective experiences that are referred to as remembering and knowing in the memory literature [32]. This example suggests that studies on implicit learning and memory should involve more sensitive methods-methods that allow us to disentangle conscious from unconscious processing within a single task. The 'contamination problem' is also problematic in brain imaging studies that attempt to identify the cerebral correlates of explicit and implicit processes, for many such studies have relied on identifying discrete regions involved in either process.

In this study, we addressed this issue by taking as a starting point that processing in any task always involves both conscious and unconscious components. To identify the brain regions that are exclusively involved in the conscious component of performance, we adapted the Process Dissociation Procedure (PDP) [18] to the study of sequence learning. The PDP is a methodological framework that makes it possible to assess the relative contributions of implicit and explicit memory in any given single task. In this framework, consciousness is related to controlled responding: it is assumed that conscious knowledge should allow control on behavior, while implicit knowledge influences behavior in an automatic way. Performance is compared in two conditions where conscious (C) and unconscious (U) processes either both contribute to improve performance (Inclusion condition), or act in opposition to each other (Exclusion condition). In our study, after completing the SRT task, participants were first instructed to reproduce the training sequence or, failing recollection, to guess the location of the next stimulus (Inclusion condition). In the subsequent Exclusion condition, participants were instructed to avoid, in each trial, giving the response that would reproduce a part of the training sequence. During the Inclusion condition, generation scores reflect the contribution of both conscious (i.e., recollection) and unconscious (i.e., guessing) processes to performance $(\mathrm{C}+\mathrm{U})$. Consistently, previous reports have indicated that participants can reproduce the training sequence even when they believe that they are guessing [35]. In contrast, during the Exclusion condition, generation scores indicate failure to exclude the training material. These scores can thus be interpreted as selectively reflecting unconscious influences (U), to the extent that conscious knowledge of the sequence could only result in successful exclusion [32]. The difference between Inclusion and Exclusion scores therefore constitutes an evaluation of conscious influences on performance (C).

Based on this rationale, we conducted a PET experiment using the $\mathrm{H}_{2}{ }^{15} \mathrm{O}$ technique to reveal the brain areas that exclusively subtend the conscious component of performance during the sequence generation task. Assuming that regional cerebral blood flow $(\mathrm{rCBF})$ variations relate to the performance as estimated by the generation scores, we looked for regions where the correlation between CBF and generation scores was modulated by the generation condition (Inclusion versus Exclusion). In other words, we aimed to identify the brain areas in which the CBF increased significantly more with the Inclusion score, which reflects both explicit and implicit contributions to performance $(\mathrm{C}+\mathrm{U})$, than with the Exclusion score, which only reflects implicit contributions (U). The brain areas in which an interaction effect is observed should therefore be specifically and exclusively involved in subtending the conscious component of performance.

\section{Materials and methods}

This study was approved by the Ethics Committee of the Faculty of Medicine of the University of Liege. Subjects were 17 young (range 18-32 years), healthy, right-handed males, recruited through advertisement. Written informed consent was obtained from all subjects.

Before the SRT task, participants were simply told that the goal of the experiment was to study, with PET, the cerebral effects of sustained practice on a simple motor task. On each trial of the four-choice SRT task, a black circle appeared on a computer screen $2 \mathrm{~cm}$ above one out of four permanent black squares spaced $4 \mathrm{~cm}$ apart. Participants (using the right hand) were instructed to press

Other measurement models have been proposed to estimate unconscious influences on behavior. In the context of a generation task, however, exclusion scores constitute the appropriate measure of implicit knowledge influence. 
as fast and as accurately as possible on the key corresponding to the location of the target on the screen. The target was erased after each response and appeared at another location after a $250 \mathrm{~ms}$ delay. Errors were indicated by a short beep. A short break occurred after each block. Unknown to participants, each block contained eight repetitions of one of the following two 12-element sequences: 342312143241 (S1) or 341243142132 (S2). These sequences are identical insofar as stimulus location and transition frequency are concerned, but differ in terms of which subsequences of three elements they contain. To assess the extent to which subjects learned a trained sequence, block 13 contained the other sequence (if a participant was trained on S1, S2 was used on block 13 and vice versa). Assuming that reaction time improvement reflects motor response preparation and anticipation of the next stimulus, reaction times should increase on block 13 only if participants have acquired specific knowledge about the sequential regularities characteristic of the training sequence presented over blocks $1-12$ (as supported by numerous experiments in the sequence learning literature $[8,10,15])$. Participants practiced the SRT task lying in the scanner, but were not scanned at this time.

After completion of the SRT task, participants were told that the sequence of stimuli contained regularities, and were then introduced to the generation task, during which they were scanned in both the Inclusion and Exclusion conditions (three scans each). In both Inclusion and Exclusion conditions, each scan began with the presentation of a randomly selected stimulus. Participants then had to indicate, throughout the 90-s duration of the scan, the location of the next stimulus by pressing on the corresponding key. The stimulus moved according to participants' responses. To avoid further learning during generation, no feedback was given in this task. During the first three scans, participants were asked to perform the inclusion task, i.e. to try to reproduce the training sequence or, failing recollection, to guess the location of the next stimulus. During the next three scans, they performed the exclusion task, where they had to try, on each trial, to avoid reproducing the sequential regularities of the training sequence. In the Exclusion condition, to ensure that participants indeed performed exclusion on each trial, they were specifically instructed not to systematically repeat a sequence they believed to be different from the training one, and not to apply particular strategies such as repeating the same response throughout the task. In both tasks, participants were also instructed not to produce repetitions (which never occurred in the training material).

Regional cerebral blood flow (rCBF), taken as a marker of local synaptic activity [20], was estimated using infusions of $\mathrm{H}_{2}{ }^{15} \mathrm{O}$ during six scans obtained successively in Inclusion and Exclusion (three scans each) conditions. PET data were processed using SPM99 (Wellcome Department of Cognitive Neurology, London, UK) implemented in MATLAB (Mathworks, Sherborn, MA, USA). For each subject, all scans were realigned together. A mean PET image was created, to which the subject's MRI scan was coregistered. PET and MRI images were then normalized to a standard PET template using the same transformations. Finally, PET images were smoothed using a Gaussian kernel of $16 \mathrm{~mm}$ full width at half maximum. Scan condition (Inclusion or Exclusion), covariate of interest (generation score at each scan), condition by covariate, and subject (block) effects were estimated at each and every voxel according to the general linear model. Global flow adjustment was performed by subject-specific analysis of covariance, and the number of responses generated during each scan was entered as a confounding covariate to control for individual differences in response frequency and motor-related activations. The resulting set of voxel values for each contrast constituted a map of the t statistic $[\mathrm{SPM}(\mathrm{T})]$ thresholded at $\mathrm{P}<0.001$, uncorrected $(\mathrm{T} \leq 3.14)$ and reported descriptively in tabular format. However, the discussion is restricted to results that survived brain volume correction for multiple comparisons thresholded at $\mathrm{P}^{\mathrm{corr}}<0.05$. Preliminary analyses estimated the task effect (Inclusion versus Exclusion scans) and the correlation between $\mathrm{rCBF}$ and generation score in each condition. The effect of interest (i.e., the brain areas that specifically subtend the conscious component of performance) was assessed using a condition (Inclusion vs. Exclusion) by rCBF correlation with performance (generation score) interaction analysis. This analysis relies on a fixed effect model. The results, therefore, only pertain to the sampled population.

\section{Results}

\subsection{Behavioral data}

Before PET image acquisition, participants were trained on a four-choice serial reaction time task during 15 blocks of 96 successive trials. Unknown to them, each block contained eight repetitions of a 12-element sequence. An analysis of variance (ANOVA) indicated that the mean reaction time (RT) for each block gradually decreased with practice $[\mathrm{F}(14,224)=15.929$, Mse $=35470, \mathrm{P}<0.0001]$. A 
significant increase of $119 \mathrm{~ms}$ was observed when a different sequence was presented during the thirteenth block [paired, one-tailed $\mathrm{t}(16)=6.41, \mathrm{P}<0.0001$; see Fig. 1]. This cost in reaction time demonstrates optimized motor preparation specific to the trained sequence, and is consistent with previous studies indicating that subjects are able to learn the sequential constraints of a structured series of stimuli $[9,36]$.

After completion of the SRT task, participants were informed that the sequence had followed a repeating pattern. They were then introduced to the sequence generation task in which they had to indicate the location at which they thought the next stimulus would appear rather than to react to the location of the current stimulus.

\section{Serial Reaction Time Task}

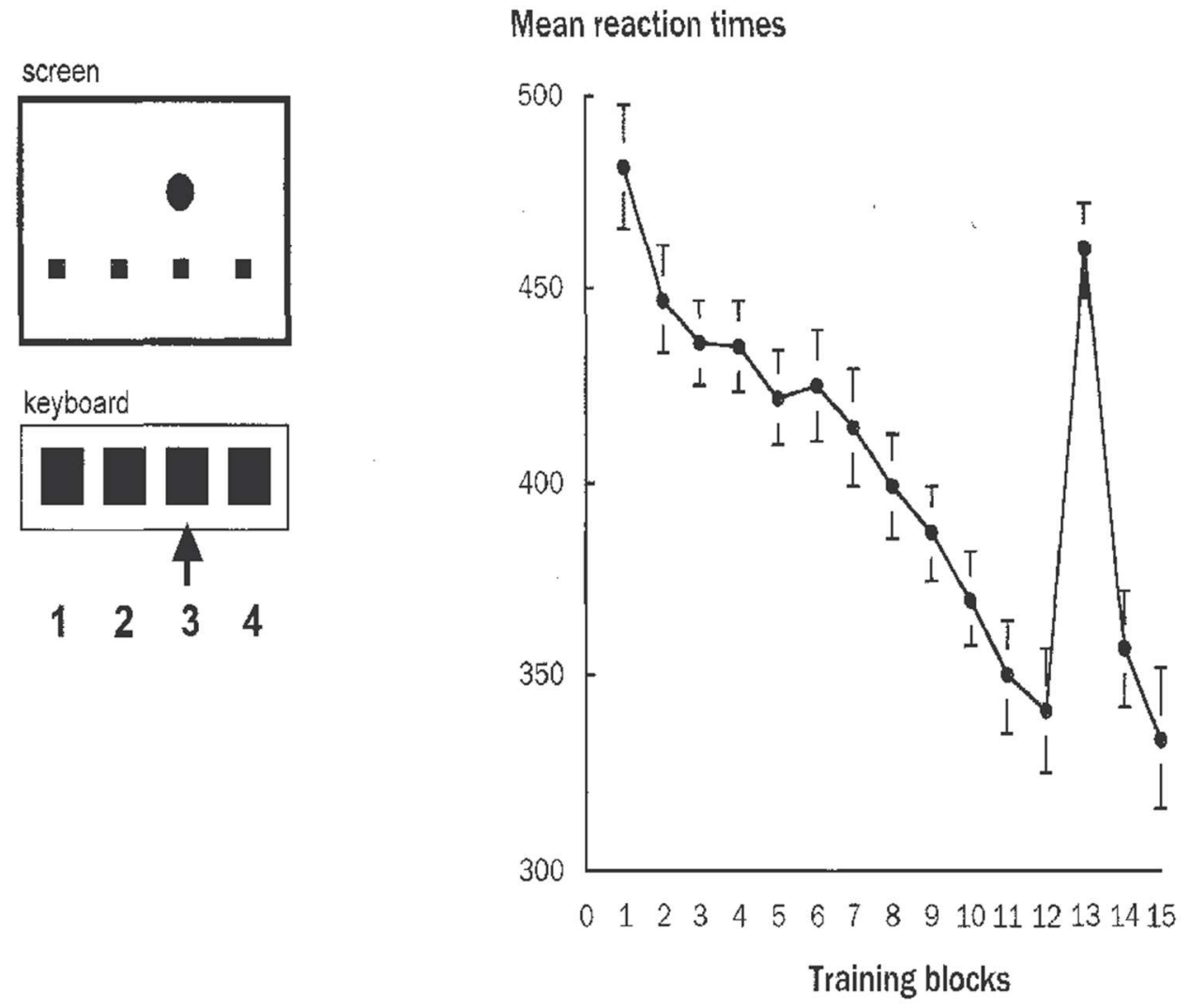

Fig. 1. Mean reaction times for each training block in the SRT task.

Recall that participants were instructed to reproduce the training sequence in the Inclusion condition, and to avoid reproducing it in the Exclusion condition. Performance was measured, for each inclusion and exclusion scan, by the number of generated chunks of three elements that were part of the training sequence, divided by the total number of triplets produced during that scan (see Table 1).

Generation scores for the three scans of each condition failed to differ from one another $(\mathrm{F}<1)$. On average, participants were more likely to produce chunks from the training sequence during Inclusion $(71 \%)$ than during Exclusion $\{31 \%[\mathrm{~F}(1,15)=41.254 ; \mathrm{P}<0.0001]\}$, suggesting that they had acquired explicit knowledge about the sequence during training.

Table 1Mean generation scores

\begin{tabular}{lll}
\hline & Inclusion & Exclusion \\
\hline Block 1 & $0.71(0.24)$ & $0.29(0.13)$ \\
Block 2 & $0.72(0.26)$ & $0.31(0.09)$ \\
Block 3 & $0.71(0.26)$ & $0.33(0.17)$ \\
\hline
\end{tabular}

Notes: Mean proportions (and standard deviations) of sequences of three elements that were part of the training sequence, generated during each block in Inclusion and Exclusion conditions. 
Response frequency, however, was significantly influenced both by Block repetition and by Instructions. The mean number of generated responses increased between the first and the third scan within each condition $[\mathrm{F}(2,30)=5.88 ; \mathrm{P}<0.01]$ and was higher during Inclusion than during Exclusion $[\mathrm{F}(1,15)=5.7 ; \mathrm{P}<0.05]$. The interaction Block X Instructions was not significant $(\mathrm{F}<1)$. To control for the effect of response frequency, the number of generated responses for each scan was used as a confounding covariate in the analysis of PET data.

\subsection{PET data}

Preliminary analyses did not reveal a significant task effect (Inclusion vs. Exclusion scans; $\mathrm{P}^{\mathrm{corr}}>0.05$ ), suggesting that instruction type (i.e., to reproduce versus to avoid reproducing the training sequence) did not significantly influence regional brain activity. At a lower statistical threshold $(\mathrm{P}<$ 0.001, uncorrected), however, several middle and superior frontal regions, as well as part of the right inferior parietal lobule, were more active in the Exclusion than in the Inclusion condition. This could reflect increased demands on attentional and monitoring resources during the exclusion task. Conversely, posterior, parahippocampal and occipital regions were more active in the Inclusion than in the Exclusion condition. These results are reported descriptively for completeness only (see Table 2) and will not be discussed further.

Note that several differences can be identified between the inclusion and exclusion tasks, which actually undermine the validity of a direct comparison between inclusion and exclusion conditions. First, as every participant performed the inclusion task before the exclusion task, time order is a confounding factor. Second, the response selection differs in both tasks as the inclusion task requires choosing one response amongst three possible successors whereas, in the exclusion task, one element must first be excluded and the response is then selected between the two remaining possibilities. Therefore, rCBF differences between conditions could possibly be related to time order or task modality effects, which explains why we were not primarily interested in these differences. Instead, our main question centered on differences in the regression of $\mathrm{rCBF}$ on the performance measure, given that generation scores reflect the contribution of conscious and unconscious (Inclusion condition) or only unconscious (Exclusion condition) processes to the performance. To identify the brain regions that are exclusively in charge of the explicit component of performance, we considered the areas where the regression between CBF and performance (generation) scores was modulated by the condition (Inclusion versus Exclusion). Such an interaction was observed in the left mesial frontal area, across the border of the anterior cingulate cortex, and the adjacent mesial prefrontal cortex (Brodmann area 32/10; coordinates [-16 $422 \mathrm{~mm}$ ]; $\mathrm{P}^{\text {corr }}<0.05$; Fig. 2). Given the structural variability of the cingulate sulcus and the variable presence of the para-cingulate gyrus [29], the exact architectonic location of this area cannot be easily defined and we refer to it as anterior cingulate/mesial prefrontal cortex (ACC/MPFC). At a lower statistical threshold ( $\mathrm{P}<0.001$, uncorrected), an interaction effect was likewise disclosed in the thalamus [6-18 $16 \mathrm{~mm}$ ] and the superior temporal gyrus (BA 22; [-50 -34 8 $\mathrm{mm}]$ ).

Regional CBF regression with generation score in each condition separately did not reach significance after correction for multiple comparisons ( $\mathrm{P}^{\mathrm{corr}}>0.05$ ). Results significant at $\mathrm{P}<0.001$ (uncorrected) are reported descriptively for completeness only (see Table 3 ).

\section{Discussion}

In this experiment, participants were first trained on a standard SRT task and then scanned during a subsequent generation task performed under inclusion and exclusion instructions.

Behavioral results show that participants learned the sequential regularities of the sequence they were presented with in the SRT task. First, reaction time increased when the training pattern was switched to another one in the transfer block of the SRT task. Second, participants were able to reproduce much of the sequential regularities in the Inclusion condition.

Was sequence learning explicit or implicit in this experiment? Participants were successful in avoiding the generation of the training material in the Exclusion condition. This result suggests that learning was essentially explicit. Nonetheless, we cannot rule out the possibility that some implicit knowledge influenced generation performance in the Inclusion and Exclusion conditions without being detected in 
the latter condition because of the limited sensitivity of the task. However, we can assume that the sequence was, at least partly, learned explicitly, since Inclusion scores-which reflect both implicit and explicit influences-were higher than Exclusion scores, which reflect implicit influences only. What are the brain regions that subtend explicit sequence processing?

Table 2 Task effect. Categorical comparisons

\begin{tabular}{|c|c|c|c|c|c|c|}
\hline \multirow{2}{*}{$\begin{array}{l}\text { Area of } \\
\text { activation }\end{array}$} & \multirow{2}{*}{$\begin{array}{l}\text { Hemisphere } \\
\text { L(eft), R(ight) }\end{array}$} & \multicolumn{5}{|c|}{ BA Coordinates Voxel } \\
\hline & & & $\mathrm{X}$ & $\mathrm{y}$ & $\mathrm{Z}$ & level ( $\mathrm{T}$ \\
\hline \multicolumn{7}{|l|}{ Exclusion vs. Inclusion } \\
\hline Anterior cingulate gyrus & $\mathrm{L}$ & 32 & -6 & 28 & 26 & 5.26 \\
\hline Middle frontal gyrus & $\mathrm{L}$ & 11 & -32 & 48 & -16 & 4.46 \\
\hline Middle frontal gyrus & $\mathrm{L}$ & 10 & -30 & 58 & -8 & 4.38 \\
\hline Superior frontal gyrus & $\mathrm{R}$ & 8 & 16 & 16 & 50 & 4.28 \\
\hline Middle frontal gyrus & $\mathrm{R}$ & 9 & 56 & 10 & 40 & 3.44 \\
\hline Middle frontal gyrus & $\mathrm{R}$ & 46 & 48 & 38 & 24 & 4.49 \\
\hline Precentral gyrus & $\mathrm{R}$ & 6 & 58 & 4 & 34 & 4.56 \\
\hline Inferior parietal & $\mathrm{R}$ & 40 & 50 & -62 & 46 & 4.18 \\
\hline \multicolumn{7}{|l|}{ Inclusion vs. Exclusion } \\
\hline Parahippocampal gyrus & $\mathrm{R}$ & 30 & 18 & -38 & -4 & 3.90 \\
\hline Middle occipital gyrus & $\mathrm{L}$ & 18 & -16 & -92 & 28 & 3.87 \\
\hline Fusiform gyrus & $\mathrm{L}$ & 19 & -40 & -70 & -6 & 3.73 \\
\hline
\end{tabular}

Notes: BA, Brodmann area. Coordinates $\mathrm{x}, \mathrm{y}, \mathrm{z}$ refer to the standard stereotaxic space. Only the most representative voxel is displayed for each area/structure. Results are displayed at $\mathrm{P}<0.001$, uncorrected, in clusters of $>15$ contiguous voxels. 


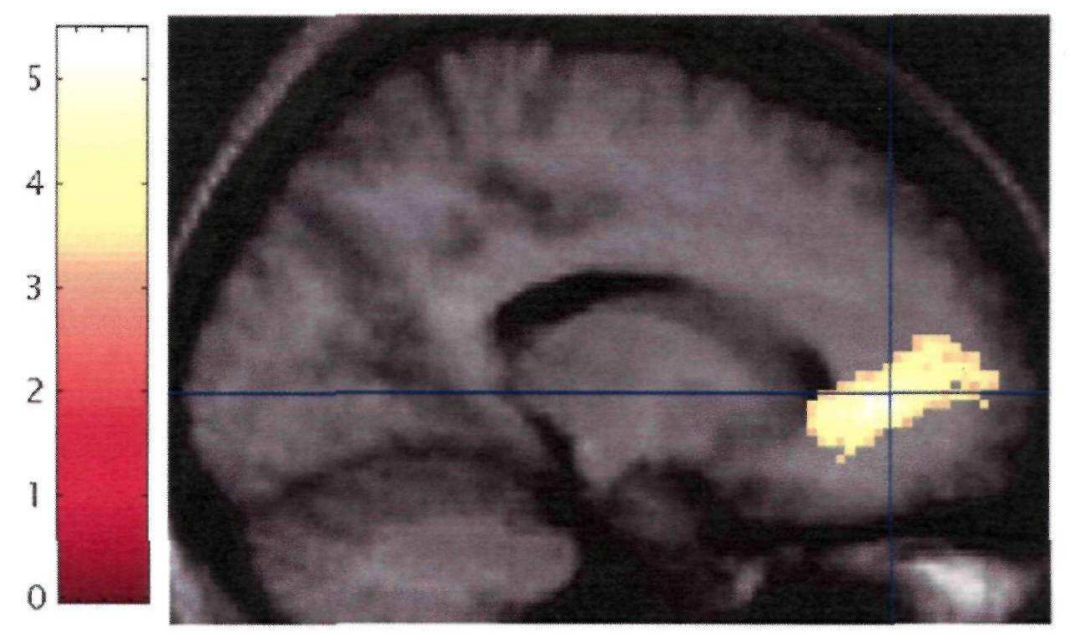

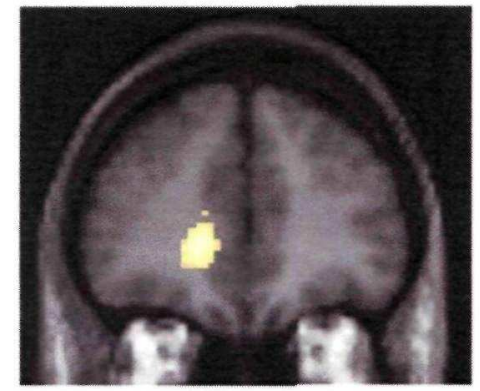

$40 \mathrm{~mm}$

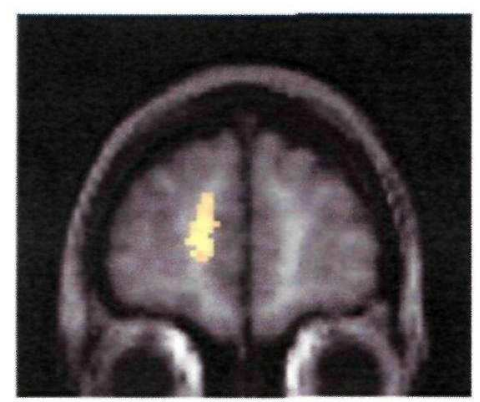

$56 \mathrm{~mm}$

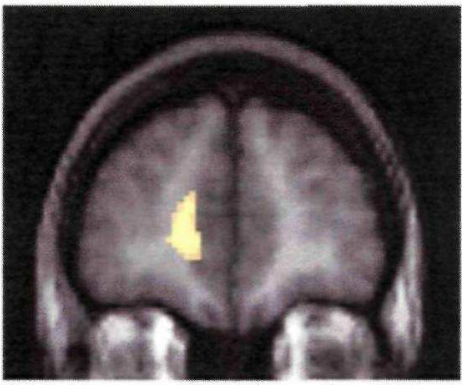

$48 \mathrm{~mm}$

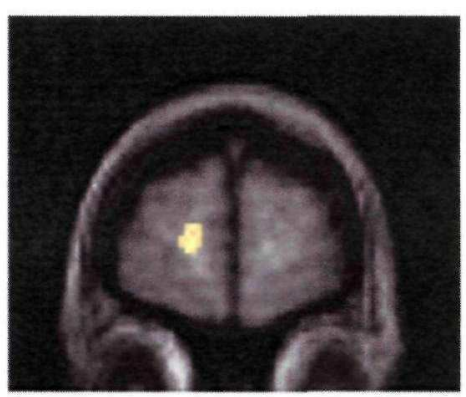

$64 \mathrm{~mm}$

Fig. 2. Brain correlates of conscious sequential knowledge. Interaction effect between condition (Inclusion versus Exclusion) and rCBF correlation with generation scores (displayed at uncorrected $\mathrm{P}<0.001$ ), on subjects' averaged Tl weighted MRL Sagittal section is $16 \mathrm{~mm}$ to the left of the interhemispheric midline. Blue lines indicate the peak voxel located in the anterior cingulate/mesial prefrontal cortex at [-16 $422 \mathrm{~mm}$ ] in standard anatomical space. Coronal sections range from 40 to $64 \mathrm{~mm}$ anterior to the commissural plane.

Our results indicate that the anterior cingulate/mesial prefrontal area is involved in this respect. Importantly, we do not assume that this region alone is responsible for sequence awareness. Other brain regions have been shown to be involved in sequence processing and likewise participate in the emergence of the trained material to consciousness, but in a way that is not exclusively related to conscious processing. Many of the different processes engaged in the sequence generation task have been shown to involve ACC/MPFC in humans: delayed processing of information [6,33], recognition of learned movements to imitate [16], sequence expectation [21], action planning and performance monitoring [3,4,24,25], decision making [17], or error processing [27]. Note that ACC is a large region, which seems to follow a functional topography, with the motor area at the back, followed by the cognitive area then the emotional regions [9]. Surprisingly, the activation peak in the present study [-16 $422 \mathrm{~mm}$ ] falls near the affective portion of the ACC, anterior and inferior to the cognitive part in which the highest activation peaks are found in most of the aforementioned studies. However, the functional topography of the ACC is not absolute and activations related to cognitive 
processes were also observed in so-called affective areas [2,7].

Our study was not designed to elucidate the processes subtending sequence generation, but rather to specifically identify those regions involved in the conscious recollection of sequential material.

Table 3 rCBF correlation with generation score in each condition. Parametric analyses

\begin{tabular}{llll}
\hline $\begin{array}{l}\text { Area of } \\
\text { activation }\end{array}$ & Hemisphere L(eft), R(ight) BA & Coordinates & Voxel level (T) \\
\cline { 2 - 4 } $\mathrm{z}$ &
\end{tabular}

\begin{tabular}{|c|c|c|c|c|c|c|}
\hline \multicolumn{7}{|l|}{ Exclusion condition ${ }^{\mathrm{a}}$} \\
\hline \multicolumn{7}{|l|}{ Anterior cingulate/medial } \\
\hline Medial frontal gyrus & $\mathrm{L}$ & 10 & -12 & 52 & 10 & 4.42 \\
\hline Caudate nucleus & $\mathrm{R}$ & $\mathrm{CN}$ & 18 & 14 & 16 & 3.91 \\
\hline Thalamus & $\mathrm{R}$ & $\mathrm{Th}$ & 2 & -24 & 14 & 3.90 \\
\hline \multicolumn{7}{|l|}{ Inclusion condition } \\
\hline Caudate nucleus & $\mathrm{L}$ & $\mathrm{CN}$ & -16 & 28 & -2 & 4.88 \\
\hline Inferior frontal gyrus & $\mathrm{L}$ & 47 & -18 & 28 & -12 & 4.18 \\
\hline Inferior frontal gyrus & $\mathrm{L}$ & 45 & -52 & 20 & 16 & 4.76 \\
\hline Inferior frontal gyrus & $\mathrm{L}$ & 10 & -54 & 40 & -2 & 3.77 \\
\hline Middle temporal gyrus & $\mathrm{L}$ & 22 & -50 & -34 & 4 & 4.32 \\
\hline Superior temporal gyrus & $\mathrm{L}$ & 42 & -60 & -28 & 14 & 4.12 \\
\hline Posterior cingulate gyrus & $\mathrm{L}$ & 31 & -22 & -62 & 10 & 3.89 \\
\hline Precentral gyrus & $\mathrm{L}$ & 44 & -56 & 6 & 12 & 4.82 \\
\hline Precuneus & $\mathrm{L}$ & 7 & -6 & -64 & 52 & 4.40 \\
\hline Thalamus & $\mathrm{R}$ & $\mathrm{Th}$ & 8 & -14 & 16 & 4.13 \\
\hline Postcentral gyrus & $\mathrm{R}$ & 5 & 8 & -44 & 66 & 3.72 \\
\hline Precuneus & $\mathrm{R}$ & 7 & 22 & -62 & 26 & 3.74 \\
\hline
\end{tabular}

Notes: BA, Brodmann area; CN, caudate nucleus; Th, thalamus. Coordinates $\mathrm{x}, \mathrm{y}, \mathrm{z}$ refer to standard stereotaxic space. Only the most representative voxel is displayed for each area/structure. Results are displayed at $\mathrm{P}<0.001$, uncorrected, in cluster of $>15$ contiguous voxels.

a For the Exclusion condition, note that we seek a negative correlation between rCBF and generation score. Indeed, successful exclusion performance is reflected by a high degree of avoidance of generating the known sequence, and therefore the lower the generation score (i.e., the number of generated chunks that were part of the training sequence) in this condition, the better is the exclusion performance.

Our findings indicate that ACC/MPFC activation is specifically involved in this aspect of performance. Previous studies have associated explicit sequence learning with activation in primary visual and inferior parietal areas [30] or identified inferior parietal, temporal, premotor and anterior cingulate activation as correlates of consciousness in sequence learning [15]. Direct comparison with our experiment is rather hazardous since, in contrast with these previous studies, participants were not scanned during the SRT task but during the generation task. Moreover, these studies were based on the assumption that explicit and implicit processes can be dissociated in two different experimental conditions. It is therefore possible that at least part of the brain areas associated with explicit learning in these studies were not exclusively related to conscious processing. At variance, we assumed that both processes contribute to performance in any task, and proposed a methodology that makes it possible to isolate the brain regions exclusively associated with explicit processing. The detailed mechanisms underpinning conscious processes are beyond the scope of this study and might involve various putative mechanisms $[11,23,38,39]$. Importantly, neuropsychological and brain imaging studies suggest that the contribution of ACC/MPFC to conscious processes is not limited to sequential material. Bilateral lesions of mesial prefrontal and cingulate cortices can lead to akinetic mutism, a disturbance of consciousness characterized by the poverty or absence of voluntary vocal, verbal or limb movements, despite apparently preserved vigilance [5,26,28]. In normal subjects, ACC and/or MPFC activation have been associated with conscious processing of emotional experience [22], category learning with awareness [1], and conscious recognition of objects learned in the recent past [34]. It is also claimed that the ACC/MPFC is involved in explicit representation of mental states of the self [13]. Consistently, our study strongly suggests that the ACC/MPFC is specifically involved in the 
conscious processing of sequential knowledge.

We conclude that the exploration of the neural correlates of consciousness should further benefit from the application of sensitive behavioral methods that enable us to overcome the limitations of task-based dissociation paradigms, so as to focus instead on the differences between the conscious and unconscious processes jointly engaged by any task.

\section{Acknowledgements}

$\mathrm{AD}$ and SL are Postdoctoral Researchers with the Fonds National de la Recherche Scientifique (FNRS; Belgium). PM and AC are respectively Senior Research Associate and Research Associate with the FNRS. PP was supported by PAI/IAP P05/04 and FMRE. This research was supported by FNRS grant number 3.4537.00, by the Reine Elisabeth Medical foundation, by special funds for scientific research of the Universite de Liège, by grant HPRN-CT-1999-00065 from the European Commission, and by a grant from the Universite Libre de Bruxelles to AC in support of PAI/IAP P4/19.

\section{References}

[1] H.J. Aizenstein, A.W. MacDonald, V.A. Stenger, R.D. Nebes, J.K. Larson, S. Ursu, C.S. Carter, Complementary category learning systems identified using event-related functional MRI, J. Cogn. Neurosci. 12 (2000) 977-987.

[2] D.M. Barch, T.S. Braver, E. Akbudak, T. Conturo, J. Ollinger, A. Snyder, Anterior cingulate cortex and response conflict: effects of response modality and processing domain, Cereb. Cortex 11 (2001) 837-848.

[3] K.F. Berman, C. Randolph, J.M. Gold, J.M. Ostrem, R. Coppola, R.E. Carson, P. Herscovitch, D.R. Weinberger, Activation of a cortical network during performance of the Wisconsin Card Sorting Test: a positron emission tomography study, Neuropsychologia 33 (1995) 1027-1046.

[4] M. Botvinick, L.E. Nystrom, K. Fissell, C.S. Carter, J.D. Cohen, Conflict monitoring versus selection-for-action in anterior cingulate cortex, Nature 402 (1999) 179-181.

[5] A. Buge, R. Escourolle, G. Rancurel, M. Poisson, Akinetic mutism and bicingular softening: three anatomo-clinical cases, Rev. Neurol. (Paris) 131 (1975) 121-131.

[6] P.W. Burgess, E. Veitch, A. de Lacy Costello, T. Shallice, The

cognitive and neuroanatomical correlates of multitasking, Neuropsychologia 38 (2000) 848-863.

[7] G. Bush, P. Luu, M.I. Posner, Cognitive and emotional influences in anterior cingulate cortex, Trends Cogn. Sci. 4 (2000)

215-222.

[8] A. Cleeremans, A. Destrebecqz, M. Boyer, Implicit learning: news from the front, Trends Cogn. Sci. 2 (1998) $406-416$.

[9] A. Cleeremans, J.L. McClelland, Learning the structure of event sequences, J. Exp. Psychol. Gen. 120 (1991) $235-253$.

[10] B.A. Clegg, G.J. DiGirolamo, S.W. Keele, Sequence learning, Trends Cogn. Sci. 2 (1998)275-281.

[11] S. Dehaene, L. Naccache, Towards a cognitive neuroscience of consciousness: basic evidence and a workspace framework, Cognition 79 (2001) 1-37.

[12] A. Destrebecqz, A. Cleeremans, Can sequence learning be implicit? New evidence with the process dissociation procedure, Psychon. Bull. Rev. 8 (2001) 343-350.

[13] CD. Frith, U. Frith, Interacting minds-A biological basis, Science 286 (1999) 1692-1695.

[14] C. Frith, R. Perry, E. Lumer, The neural correlates of conscious experience: an experimental framework, Trends Cogn. Sci. 3 (1999) 105-114.

[15] S.T. Grafton, E. Hazeltine, R. Ivry, Functional mapping of sequence learning in normal humans, J. Cogn. Neurosci. 7 (1995) 497-510.

[16] J. Grezes, N. Costes, J. Decety, The effects of learning and memory on the neural network involved in the perception of meaningless action, Brain 122 (1999) 1875-1887.

[17] A. Ikeda, H.O. Luders, T.F. Collura, R.C. Burgess, H.H. Morris, T. Hamano, H. Shibasaki, Subdural potentials at orbitofrontal and mesial prefrontal areas accompanying anticipation and decisionmaking in humans: a comparison with Bereitschaftspotential, Elec-troencephalogr. Clin. Neurophysiol. 98 (1996) 206-212.

[18] L.L. Jacoby, A process dissociation framework: separating automatic from intentional uses of memory, J. Mem. Lang. 30 (1991)

513-541.

[19] L.L. Jacoby, V. Woloshyn, C. Kelley, Becoming famous without being recognized: unconscious influence of memory produced by divided attention, J. Exp. Psychol. Gen. 118 (1989) 115-125.

[20] M. Jueptner, C. Weiller, Does measurement of regional cerebral blood flow reflect synaptic activity? Implications for PET and fMRI, Neuroimage 2 (1995) 148-156.

[21] E. Koechlin, G. Corrado, P. Pietrini, J. Grafman, Dissociating the role of the medial and lateral anterior prefrontal cortex in human planning, Proc. Natl. Acad. Sci. USA 97 (2000) 7651-7656.

[22] R.D. Lane, Neural correlates of levels of emotional awareness. Evidence of an interaction between emotion and attention in the anterior cingulate cortex, J. Cogn. Neurosci. 10 (1998) 525-535.

[23] R. Llinas, U. Ribary, D. Contreras, C. Pedroarena, The neuronal basis for consciousness, Philos. Trans. R. Soc. Lond. B: Biol. Sci. 353 (1998) 1841-1849.

[24] P. Luu, T. Flaisch, D.M. Tucker, Medial frontal cortex in action monitoring, J. Neurosci. 20 (2000) 464-469.

[25] A.W. MacDonald, J.D. Cohen, V.A. Stenger, C.S. Carter, Dissociating the role of the dorsolateral prefrontal and anterior cingulate cortex in cognitive control, Science 288 (2001) 1835-1838.

[26] M.S. Mega, R.C. Cohenour, Akinetic mutism: disconnection of frontal-subcortical circuits, Neuropsychiatry Neuropsychol. Behav. Neurol. 10 (1997) 254-259, 
[27] V. Menon, N.E. Adleman, CD. White, G.H. Glover, A.L. Reiss, Error-related brain activation during a Go/No Go response inhibition task, Hum. Brain Mapp. 12 (2001) 131-143.

[28] G. Nemeth, K. Hegediis, L. Molnar, Akinetic mutism associated with bicingular lesions. Clinicopathological and functional anatomical correlates, Eur. Arch. Psychiatry Clin. Neurosci. 237 (1998) 218-222.

[29] T. Paus, F. Tomaiuolo, N. Otaky, D. MacDonald, M. Petrides, J. Atlas, R. Morris, A.C Evans, Human cingulate and paracingulate sulci: pattern, variability, asymmetry, and probabilistic map, Cereb. Cortex 6 (1996) 207-214.

[30] S.L. Rauch, C.R. Savage, H.D. Brown, T. Curran, N.M. Alpert, A. Kendrick, A.J. Fischman, S.M. Kosslyn, A PET investigation of implicit and explicit sequence learning, Hum. Brain Mapp. 3 (1995) 271-286.

[31] J. Reed, P. Johnson, Assessing implicit learning with indirect tests: determining what is learned about sequence structure, J. Exp. Psychol. Learn. Mem. Cogn. 20 (1994) 585-594.

[32] A. Richardson-Klavehn, J.M. Gardiner, R.I. Java, Memory: task dissociations, process dissociations, and dissociation of consciousness, in: G. Underwood (Ed.), Implicit Cognition, Oxford University Press, Oxford, 1996, pp. 85-158.

[33] K. Rubia, S. Overmeyer, E. Taylor, M. Brammer, S. Williams, A. Simmons, C Andrew, E.T, Bullmore, Prefrontal involvement in temporal bridging and timing movement, Neuropsychologia 36 (1998) 1283-1293.

[34] TV. Sewards, M.A. Sewards, On the neural correlates of object recognition awareness: relationship to computational activities and activities mediating perceptual awareness, Conscious. Cogn. 11 (2002) 51-77.

[35] D.R. Shanks, T. Johnstone, Implicit knowledge in sequential learning tasks, in: M.A. Stadler, P.A. Frensch (Eds.), Handbook of Implicit Learning, Sage Publications, 1998, pp. 533-572.

[36] D.R. Shanks, T. Johnstone, Evaluating the relationship between explicit and implicit knowledge in a serial reaction time task, J. Exp. Psychol. Learn. Mem. Cogn. 25 (1999) 1435-1451.

[37] D.R. Shanks, M.F. St. John, Characteristics of dissociable human learning systems, Behav. Brain Sci. 17 (1994)367-447.

[38] W. Singer, Consciousness and the structure of neuronal representation, Philos. Trans. R. Soc. Lond. B: Biol. Sci. 353 (1998) $1829-1840$.

[39] G. Tononi, G.M. Edelman, Consciousness and complexity, Science 282 (1998) 1846-1851. 